CU-TP-1149

hep-th/0606141

\title{
Holographic representation of local bulk operators
}

\author{
Alex Hamilton ${ }^{1}$, Daniel Kabat ${ }^{1}$, Gilad Lifschytz ${ }^{2}$, \\ David A. Lowe ${ }^{3}$ \\ ${ }^{1}$ Department of Physics \\ Columbia University, New York, NY 10027 USA \\ hamilton@phys.columbia.edu, kabat@phys.columbia.edu \\ ${ }^{2}$ Department of Mathematics and Physics and CCMSC \\ University of Haifa at Oranim, Tivon 36006 ISRAEL \\ giladl@research.haifa.ac.il \\ ${ }^{3}$ Department of Physics \\ Brown University, Providence, RI 02912 USA \\ lowe@brown.edu
}

The Lorentzian AdS/CFT correspondence implies a map between local operators in supergravity and non-local operators in the CFT. By explicit computation we construct CFT operators which are dual to local bulk fields in the semiclassical limit. The computation is done for general dimension in global, Poincaré and Rindler coordinates. We find that the CFT operators can be taken to have compact support in a region of the complexified boundary whose size is set by the bulk radial position. We show that at finite $N$ the number of independent commuting operators localized within a bulk volume saturates the holographic bound. 


\section{Introduction}

The anti-de Sitter/conformal field theory (AdS/CFT) correspondence [1, 2, 3, 4, in its Lorentzian version [5, 6, 7], states that any bulk excitation is encoded on the boundary by some CFT operator or state. In the semiclassical limit of large $N$ and large 't Hooft coupling we expect to have free local fields in the bulk. These bulk fields should be encoded in the CFT. To see how this works consider a bulk field with normalizable fall-off near the boundary of AdS.

$$
\phi(z, x) \sim z^{\Delta} \phi_{0}(x)
$$

Here $z$ is a radial coordinate which vanishes at the boundary. The bulk supergravity field can be expressed in terms of the boundary field $\phi_{0}$ via a kernel $K$.

$$
\phi(z, x)=\int d x^{\prime} K\left(x^{\prime} \mid z, x\right) \phi_{0}\left(x^{\prime}\right)
$$

We will refer to $K$ as a smearing function. $\phi_{0}(x)$ corresponds to a local operator $\mathcal{O}(x)$ in the CFT [8].

$$
\phi_{0}(x) \leftrightarrow \mathcal{O}(x)
$$

Thus the AdS/CFT correspondence implies that local bulk fields are dual to non-local boundary operators [6, 9, 10, ${ }^{1}$

$$
\phi(z, x) \leftrightarrow \int d x^{\prime} K\left(x^{\prime} \mid z, x\right) \mathcal{O}\left(x^{\prime}\right)
$$

Bulk-to-bulk correlation functions, for example, are equal to correlation functions of the corresponding non-local operators in the CFT.

$\left\langle\phi\left(z_{1}, x_{1}\right) \phi\left(z_{2}, x_{2}\right)\right\rangle_{S U G R A}=\int d x_{1}^{\prime} d x_{2}^{\prime} K\left(x_{1}^{\prime} \mid z_{1}, x_{1}\right) K\left(x_{2}^{\prime} \mid z_{2}, x_{2}\right)\left\langle\mathcal{O}\left(x_{1}^{\prime}\right) \mathcal{O}\left(x_{2}^{\prime}\right)\right\rangle_{C F T}$

Smearing functions are central to understanding Lorentzian AdS/CFT: they define the map by which, in the semiclassical limit, local bulk excitations are encoded on the boundary. The semiclassical limit tightly constrains behavior at finite $N$. For example, as we will see, smearing functions can be used to count the number of independent commuting operators inside a

\footnotetext{
${ }^{1}$ For a different approach see [11, 12].
} 
volume in the bulk, even at finite $N$. They can also be used to study bulk locality and causality: for example in [13] we used them to understand the causal structure of a black hole from the boundary point of view.

The main purpose of this paper is to compute smearing functions in various pure AdS geometries. This continues the study started in 13, where a two-dimensional AdS spacetime was considered. In the present paper we extend the analysis to higher dimensions and compute smearing functions for global $\mathrm{AdS}_{d+1}$, for the Poincaré patch in $d+1$ dimensions and for $\mathrm{AdS}_{3}$ in Rindler coordinates.

It's important to recognize that smearing functions are not necessarily unique. In some cases the boundary fields do not involve a complete set of Fourier modes and we are free to add to the smearing function terms that integrate to zero against all boundary fields. This freedom enables us to present the smearing function in different forms, which is useful depending on which aspect one wishes to study.

For the impatient reader, let us briefly summarize our main results. In global coordinates we find that the smearing function can be chosen to have support on boundary points that are spacelike separated from the bulk point. This is illustrated in figure 1] The exact form of the smearing function depends on the dimension: for even-dimensional AdS it's given in (20), for odd-dimensional AdS it's given in (33).

We also construct smearing functions in the Poincaré patch. For evendimensional AdS we find that the smearing function can be taken to have support at spacelike separation in the Poincaré patch: see (35). For odddimensional AdS the smearing function has support on the entire Poincaré boundary: see (39). An alternate form of the $\mathrm{AdS}_{3}$ Poincaré smearing function is given in (83).

In Rindler coordinates we show that to construct a smearing function we must analytically continue the spatial coordinates of the boundary theory to imaginary values. This enables us to find a smearing function with support on a compact region of the complexified geometry. The explicit result for $\mathrm{AdS}_{3}$ is given in (54).

It is desirable to work with smearing functions that make the boundary operators as sharply-localized as possible. Our strongest results in this di- 


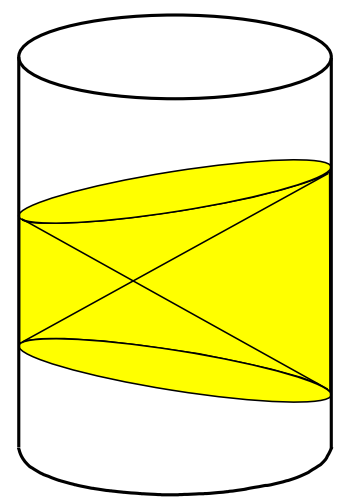

Figure 1: In global coordinates AdS resembles an infinite cylinder. We've drawn the light cones emanating from a bulk point and intersecting the boundary. The CFT operator has support on the strip indicated in yellow, at spacelike separation from the bulk point.

rection are achieved in Rindler coordinates, where we find operators with compact support on the complexified boundary. This enables us to obtain an improved understanding of bulk causality. Furthermore the statement of scale-radius duality [14, 15] can be made in a sharper way, since the size of the smeared operator is determined by the radial position of the bulk point. We would also like to stress that, as in 13, lightcone singularities of bulk correlators arise from UV singularities of the boundary theory. This is true even for bulk points deep inside AdS. Thus regions deep inside the bulk cannot be associated with a boundary theory with a conventional UV cutoff.

An outline of this paper is as follows. In section 2 we compute the smearing function in global coordinates. In section [3 we compute the smearing function in Poincaré coordinates. In section 4 we compute the smearing function for $\mathrm{AdS}_{3}$ in Rindler coordinates, and in section 5 we discuss some of the implications of our results for bulk locality and holography. Finally appendix A presents an alternate derivation of the global smearing function in even-dimensional AdS, appendix B shows that the smearing functions are AdS-covariant, and appendix $\mathrm{C}$ presents an alternate derivation of the Poincaré smearing function in $\mathrm{AdS}_{3}$. 


\section{Global AdS}

In this section we construct smearing functions in global coordinates. The construction is based on mode sums. We treat even-dimensional AdS in section 2.2 and odd-dimensional AdS in section 2.3. In appendix $\mathrm{A}$ we present an alternate approach to the even-AdS global smearing function, where the construction is based on a Greens function.

\section{$2.1 \quad$ Preliminaries}

We will describe $\operatorname{AdS}_{D=d+1}$ in global coordinates

$$
d s^{2}=\frac{R^{2}}{\cos ^{2} \rho}\left(-d \tau^{2}+d \rho^{2}+\sin ^{2} \rho d \Omega_{d-1}^{2}\right)
$$

where $R$ is the AdS radius, $-\infty<\tau<\infty, 0 \leq \rho<\pi / 2$, and $d \Omega_{d-1}^{2}$ is the metric on a unit $(d-1)$-sphere. An AdS-invariant distance function is given by

$$
\sigma\left(x \mid x^{\prime}\right)=\frac{\cos \left(\tau-\tau^{\prime}\right)-\sin \rho \sin \rho^{\prime} \cos \left(\Omega-\Omega^{\prime}\right)}{\cos \rho \cos \rho^{\prime}}
$$

where $\Omega-\Omega^{\prime}$ is the angular separation on the sphere. For $-\pi<\tau-\tau^{\prime}<\pi$ points with $\sigma>1$ are spacelike separated, while points with $\sigma=1$ are lightlike separated and points with $\sigma<1$ are timelike separated (although they can only be connected by a timelike geodesic if $-1<\sigma<1$ ). Solutions to the wave equation $\left(\square-m^{2}\right) \phi=0$ can be expanded in normalizeable modes

$\phi(\tau, \rho, \Omega)=\sum_{n=0}^{\infty} \sum_{l, m} a_{n l m} e^{-i(2 n+l+\Delta) \tau} \cos ^{\Delta} \rho \sin ^{l} \rho P_{n}^{\left(\Delta-\frac{d}{2}, l+\frac{d}{2}-1\right)}(-\cos 2 \rho) Y_{l m}(\Omega)+$ c.c.

where $P_{n}^{(\alpha, \beta)}$ is a Jacobi polynomial, $Y_{l m}$ is a spherical harmonic, and the

conformal dimension of the corresponding operator is $\Delta=\frac{d}{2}+\sqrt{\frac{d^{2}}{4}+m^{2} R^{2}}$. In global coordinates we define the boundary value of the field

$$
\phi_{0}^{\text {global }}(\tau, \Omega)=\lim _{\rho \rightarrow \pi / 2} \frac{\phi(\tau, \rho, \Omega)}{\cos ^{\Delta} \rho} .
$$




\subsection{Even AdS: mode sum approach}

We assume that $D$ is even and construct a smearing function starting from the mode expansion (44). We first work at the center of AdS (meaning $\rho=0$ ), where only the $s$-wave contributes, and later extend our results to arbitrary bulk points. At the center

$$
\phi(\tau, \rho=0, \Omega)=\sum_{n=0}^{\infty} a_{n} e^{-i(2 n+\Delta) \tau} P_{n}^{\left(\Delta-\frac{d}{2}, \frac{d}{2}-1\right)}(-1)+\text { c.c. }
$$

We can split the corresponding $s$-wave part of the boundary field into its positive and negative frequency components,

$$
\begin{aligned}
& \phi_{0}^{\text {global }}(\tau)=\phi_{0+}^{\text {global }}(\tau)+\phi_{0-}^{\text {global }}(\tau) \\
& \phi_{0+}^{\text {global }}=\sum_{n=0}^{\infty} a_{n} e^{-i(2 n+\Delta) \tau} P_{n}^{\left(\Delta-\frac{d}{2}, \frac{d}{2}-1\right)}(1) \\
& \phi_{0-}^{\text {global }}=\sum_{n=0}^{\infty} a_{n}^{*} e^{i(2 n+\Delta) \tau} P_{n}^{\left(\Delta-\frac{d}{2}, \frac{d}{2}-1\right)}(1) .
\end{aligned}
$$

Note that

$$
a_{n}=\frac{1}{\pi \operatorname{vol}\left(S^{d-1}\right) P_{n}^{\left(\Delta-\frac{d}{2}, \frac{d}{2}-1\right)}(1)} \int_{-\pi / 2}^{\pi / 2} d \tau \int d \Omega \sqrt{g_{\Omega}} e^{i(2 n+\Delta) \tau} \phi_{0+}^{\text {global }}(\tau) .
$$

Plugging this back into the bulk mode expansion (6) we can write the field at the origin of AdS (meaning the point $\tau^{\prime}=\rho^{\prime}=0$ ) as

$$
\left.\phi\right|_{\text {origin }}=\int_{-\pi / 2}^{\pi / 2} d \tau \int d \Omega \sqrt{g_{\Omega}} K_{+}\left(\tau, \Omega \mid \tau^{\prime}, \rho^{\prime}, \Omega^{\prime}\right) \phi_{0+}^{\text {global }}(\tau, \Omega)+\text { c.c. }
$$

where

$$
K_{+}=\frac{1}{\pi \operatorname{vol}\left(S^{d-1}\right)} \sum_{n=0}^{\infty} e^{i(2 n+\Delta) \tau} \frac{P_{n}^{\left(\Delta-\frac{d}{2}, \frac{d}{2}-1\right)}(-1)}{P_{n}^{\left(\Delta-\frac{d}{2}, \frac{d}{2}-1\right)}(1)} .
$$

The sum can be evaluated as

$$
\begin{aligned}
K_{+} & =\frac{\Gamma\left(\Delta-\frac{d}{2}+1\right)}{\pi \operatorname{vol}\left(S^{d-1}\right) \Gamma(d / 2)} e^{i \Delta \tau} \sum_{n=0}^{\infty} \frac{\Gamma\left(n+\frac{d}{2}\right)}{\Gamma\left(n+\Delta-\frac{d}{2}+1\right)}\left(-e^{i 2 \tau}\right)^{n} \\
& =\frac{1}{\pi \operatorname{vol}\left(S^{d-1}\right)} e^{i \Delta \tau} F\left(1, \frac{d}{2}, \Delta-\frac{d}{2}+1,-e^{i 2 \tau}\right)
\end{aligned}
$$


where strictly speaking to make the sum convergent we should have replaced $\tau \rightarrow \tau+i \epsilon$.

In terms of $z=e^{i 2 \tau}$ we have $K_{+}=\frac{1}{\pi \operatorname{vol}\left(S^{d-1}\right)} z^{\Delta / 2} F\left(1, \frac{d}{2}, \Delta-\frac{d}{2}+1,-z\right)$. At this point it's useful to make a $z \rightarrow 1 / z$ transformation of the hypergeometric function. This gives

$$
\begin{gathered}
K_{+}=\frac{z^{\Delta / 2}}{\pi \operatorname{vol}\left(S^{d-1}\right)}\left\{\frac{\Gamma\left(\Delta-\frac{d}{2}+1\right) \Gamma\left(\frac{d}{2}-1\right)}{\Gamma(d / 2) \Gamma(\Delta-d / 2)} z^{-1} F\left(1,1+\frac{d}{2}-\Delta, 2-\frac{d}{2},-\frac{1}{z}\right)\right. \\
\left.+\frac{\Gamma\left(\Delta-\frac{d}{2}+1\right) \Gamma\left(1-\frac{d}{2}\right)}{\Gamma(1) \Gamma(\Delta-d+1)} z^{-d / 2} F\left(d-\Delta, \frac{d}{2}, \frac{d}{2},-\frac{1}{z}\right)\right\} .
\end{gathered}
$$

It's important to note that smearing functions aren't unique, since we could replace

$$
K_{+} \rightarrow K_{+}+z^{\Delta / 2} \sum_{n=1}^{\infty} c_{n} z^{-n}
$$

for any set of $c_{n}$ : the extra terms involve Fourier components which are absent from the mode expansion (8), so they drop out when integrated against $\phi_{0+}^{\text {global }}$. This freedom can be used to eliminate the first line in (14), as can be seen by expanding the hypergeometric function there in powers of $1 / z .^{2}$ Then using $F(\alpha, \beta, \beta, x)=(1-x)^{-\alpha}$ in the second line of (14) we are left with

$$
K_{+}=\frac{\Gamma\left(\Delta-\frac{d}{2}+1\right) \Gamma\left(1-\frac{d}{2}\right)}{\pi \operatorname{vol}\left(S^{d-1}\right) \Gamma(\Delta-d+1)}\left(\sqrt{z}+\frac{1}{\sqrt{z}}\right)^{\Delta-d} .
$$

Note that $K_{+}$is real, so we can set $K=K_{+}=K_{-}$. The full smearing function for a bulk point at the origin is then given by

$$
K=\frac{\Gamma\left(\Delta-\frac{d}{2}+1\right) \Gamma\left(1-\frac{d}{2}\right)}{\pi \operatorname{vol}\left(S^{d-1}\right) \Gamma(\Delta-d+1)}(2 \cos \tau)^{\Delta-d} .
$$

It's useful to express this in terms of the invariant distance (31). In global coordinates the regulated distance from the origin of AdS to a point on the

\footnotetext{
${ }^{2}$ We're cheating a bit here, given our $\tau \rightarrow \tau+i \epsilon$ prescription, since the hypergeometric series only converges inside the unit disc. This can be taken into account by slightly deforming the $z$ contour of integration.
} 
boundary is $\lim _{\rho \rightarrow \pi / 2} \sigma \cos \rho=\cos \tau$. In terms of this regulated distance the smearing function for a bulk point at the origin is

$$
K=\frac{\Gamma\left(\Delta-\frac{d}{2}+1\right) \Gamma\left(1-\frac{d}{2}\right)}{\pi \operatorname{vol}\left(S^{d-1}\right) \Gamma(\Delta-d+1)} \lim _{\rho \rightarrow \pi / 2}(2 \sigma \cos \rho)^{\Delta-d} .
$$

To extend this to an arbitrary bulk point $P$ we can first use an AdS isometry to move $P$ to the origin, then apply the smearing function (18) to the transformed boundary data. Alternatively we can use the original boundary data but transform the smearing function. This is straightforward because (18) is AdS covariant. Thus for an arbitrary bulk point we have

$$
\phi(P)=\int_{-\infty}^{\infty} d \tau \int d \Omega \sqrt{g_{\Omega}} K(\tau, \Omega \mid P) \phi_{0}^{\text {global }}(\tau, \Omega)
$$

where

$$
\begin{aligned}
& K(\tau, \Omega \mid P)=c_{d \Delta} \lim _{\rho \rightarrow \pi / 2}(\sigma(x \mid P) \cos \rho)^{\Delta-d} \theta(\text { spacelike }) \\
& c_{d \Delta}=\frac{(-1)^{(d-1) / 2} 2^{\Delta-D} \Gamma\left(\Delta-\frac{d}{2}+1\right)}{\pi^{d / 2} \Gamma(\Delta-d+1)}
\end{aligned}
$$

In appendix $\mathrm{A}$ we reproduce this result by constructing a Greens function for the bulk wave equation.

\subsection{Odd AdS: mode sum approach}

We now assume that $D$ is odd. As in the previous subsection we first work at the origin of AdS (meaning $\tau=\rho=0$ ), where only the $s$-wave contributes, and later extend our results to arbitrary bulk points.

The result (13) holds in any number of dimensions, so in terms of $z=e^{i 2 \tau}$ we have the positive-frequency part of the global smearing function for a bulk point at the origin of AdS

$$
K_{+}=\frac{1}{\pi \operatorname{vol}\left(S^{d-1}\right)} z^{\Delta / 2} F\left(1, \frac{d}{2}, \Delta-\frac{d}{2}+1,-z\right) .
$$

At this point it's useful to make a $z \rightarrow 1 / z$ transformation of the hyperge-

ometric function. Noting that $\frac{d}{2}$ is an integer, the relevant formula can be 
found in [16], p. 109 equation (7). Again it's important to note that the smearing functions aren't unique, since we could replace

$$
K_{+} \rightarrow K_{+}+z^{\Delta / 2} \sum_{n=1}^{\infty} c_{n} z^{-n}
$$

for any $c_{n}$ since the extra terms drop out when integrated against $\phi_{0+}^{\text {global }}$. Making the $z \rightarrow 1 / z$ transformation and dropping terms that don't contribute we are left with

$$
K_{+}=-\frac{z^{(\Delta-d) / 2} \log z}{\pi \operatorname{vol}\left(S^{d-1}\right) \Gamma(d / 2) \Gamma(d / 2-\Delta)} \sum_{n=0}^{\infty} \frac{1}{n !} \Gamma(n+d-\Delta)(-z)^{-n}
$$

With some transformations of the gamma function, the binomial series can be rewritten as

$$
(1+x)^{\alpha}=-\frac{1}{\pi} \sin (\pi \alpha) \Gamma(\alpha+1) \sum_{n=0}^{\infty} \frac{1}{n !} \Gamma(n-\alpha)(-x)^{n} .
$$

So in fact

$$
K_{+}=\frac{(-1)^{(d-2) / 2} \Gamma\left(\Delta-\frac{d}{2}+1\right)}{2 \pi^{1+\frac{d}{2}} \Gamma(\Delta-d+1)} \lim _{\rho \rightarrow \pi / 2}(2 \sigma \cos \rho)^{\Delta-d} \log z
$$

where we introduced the invariant distance (3) from the origin of AdS to a point on the boundary via

$$
\lim _{\rho \rightarrow \pi / 2} 2 \sigma \cos \rho=\sqrt{z}+\frac{1}{\sqrt{z}} .
$$

Using (24) in (11), we can express the value of the field at the origin of AdS as

$\left.\phi\right|_{\text {origin }}=A \int_{-\pi / 2}^{\pi / 2} d \tau \int d \Omega \sqrt{g_{\Omega}} \lim _{\rho \rightarrow \pi / 2}\left(2 \sigma\left(x \mid x^{\prime}\right) \cos \rho\right)^{\Delta-d} \log z\left(\phi_{0+}^{\text {global }}(\tau, \Omega)-\phi_{0-}^{\text {global }}(\tau, \Omega)\right)$

where $A=\frac{(-1)^{(d-2) / 2} \Gamma\left(\Delta-\frac{d}{2}+1\right)}{2 \pi^{1+\frac{d}{2}} \Gamma(\Delta-d+1)}$. This is progress, but we'd like to express $\phi$ in terms of the local combination $\phi_{0}^{\text {global }}=\phi_{0+}^{\text {global }}+\phi_{0-}^{\text {global }}$. To do this it's useful to note that for even $d$

$$
\lim _{\rho \rightarrow \pi / 2}(2 \sigma \cos \rho)^{\Delta-d}=z^{\Delta / 2} z^{-d / 2}\left(1+\frac{1}{z}\right)^{\Delta-d}
$$


has an expansion in inverse powers of $z$,

$$
\lim _{\rho \rightarrow \pi / 2}(2 \sigma \cos \rho)^{\Delta-d}=z^{\Delta / 2} \sum_{n=1}^{\infty} c_{n} z^{-n} .
$$

A function of this form vanishes when integrated against $\phi_{0+}^{\text {global }}$. Likewise, by expanding in positive powers of $z$, it vanishes when integrated against $\phi_{0-}^{\text {global }}$. So we have the identity ${ }^{3}$

$$
\int_{-\pi / 2}^{\pi / 2} d \tau \int d \Omega \sqrt{g_{\Omega}} \lim _{\rho \rightarrow \pi / 2}(\sigma \cos \rho)^{\Delta-d}\left(\phi_{0+}^{\text {global }}(\tau, \Omega)+\phi_{0-}^{\text {global }}(\tau, \Omega)\right)=0 .
$$

Differentiating this identity with respect to $\Delta$, including the factors of $z^{ \pm \Delta / 2}$ hidden in the mode expansion of $\phi_{0 \mp}^{\text {global }}$, we obtain ${ }^{4}$

$$
\begin{aligned}
& \int_{-\pi / 2}^{\pi / 2} d \tau \int d \Omega \sqrt{g_{\Omega}} \lim _{\rho \rightarrow \pi / 2}(\sigma \cos \rho)^{\Delta-d} \log z\left(\phi_{0+}^{\text {global }}-\phi_{0-}^{\text {global }}\right) \\
& =2 \int_{-\pi / 2}^{\pi / 2} d \tau \int d \Omega \sqrt{g_{\Omega}} \lim _{\rho \rightarrow \pi / 2}(\sigma \cos \rho)^{\Delta-d} \log (\sigma \cos \rho) \phi_{0}^{\text {global }} .
\end{aligned}
$$

This lets us express the value of the field at the origin of AdS in terms of an integral over points on the boundary that are spacelike separated from the origin:

$$
\left.\phi\right|_{\text {origin }}=2 A \int_{-\pi / 2}^{\pi / 2} d \tau \int d \Omega \sqrt{g_{\Omega}} \lim _{\rho \rightarrow \pi / 2}(2 \sigma \cos \rho)^{\Delta-d} \log (\sigma \cos \rho) \phi_{0}^{\text {global }} .
$$

Finally we'd like to extend these results to an arbitrary bulk point. We claim that

$$
\phi\left(x^{\prime}\right)=\int_{-\infty}^{\infty} d \tau \int d \Omega \sqrt{g_{\Omega}} K\left(\tau, \Omega \mid x^{\prime}\right) \phi_{0}^{\text {global }}(\tau, \Omega)
$$

\footnotetext{
${ }^{3}$ This identity shows that a global smearing function of the form one might have naively expected, namely $K \sim(\sigma \cos \rho)^{\Delta-d}$, cannot be correct in odd-dimensional AdS.

${ }^{4}$ We differentiate with respect to $\Delta$ holding the quantities $a_{n} P_{n}^{\left(\Delta-\frac{d}{2}, \frac{d}{2}-1\right)}(1)$, $a_{n}^{*} P_{n}^{\left(\Delta-\frac{d}{2}, \frac{d}{2}-1\right)}(1)$ which appear in the mode expansions fixed. See (8), (9).
} 
where

$$
\begin{aligned}
K\left(\tau, \Omega \mid x^{\prime}\right) & =a_{d \Delta} \lim _{\rho \rightarrow \pi / 2}\left(\sigma\left(x \mid x^{\prime}\right) \cos \rho\right)^{\Delta-d} \log \left(\sigma\left(x \mid x^{\prime}\right) \cos \rho\right) \theta \text { (spacelike) } \\
a_{d \Delta} & =\frac{(-1)^{(d-2) / 2} 2^{\Delta-d} \Gamma\left(\Delta-\frac{d}{2}+1\right)}{\pi^{1+\frac{d}{2}} \Gamma(\Delta-d+1)}
\end{aligned}
$$

The argument is as follows. To compute the field at $x^{\prime}$ one can first use an AdS isometry to move $x^{\prime}$ to the origin, then use the smearing function (31) to compute $\phi$ at the origin in terms of the transformed boundary data. Equivalently, one can use the original boundary data but transform the smearing function. This is easy to do because, as we show in appendix B, (32) is secretly AdS covariant.

\section{Poincaré smearing}

In Poincaré coordinates the AdS metric is

$$
d s^{2}=\frac{R^{2}}{Z^{2}}\left(-d T^{2}+|d \vec{X}|^{2}+d Z^{2}\right)
$$

where $R$ is the AdS radius and $0<Z<\infty$. These coordinates cover a wedgeshaped region of global AdS. In section 3.1 we work in even-dimensional AdS and construct a spacelike smearing function with support in the Poincaré patch, starting from our global result (20). In section 3.2 we follow the same procedure in odd-dimensional AdS, and find that for generic $\Delta$ it leads to a smearing function with support on the entire Poincaré boundary. In appendix $\mathrm{C}$ we present an alternate form of the smearing function for $\mathrm{AdS}_{3}$, based on mode sums in the Poincaré patch.

\subsection{Even AdS}

In even AdS we can construct a spacelike smearing function with support in a Poincaré patch, starting from the global spacelike smearing function (20). 
In global coordinates the antipodal map acts via ${ }^{5}$

$$
A: \tau \rightarrow \tau \pm \pi, \quad \rho \text { invariant }, \quad \Omega \rightarrow \Omega_{A}
$$

where $\Omega_{A}$ is the antipodal point on the sphere. The positive-frequency part of a bulk field transforms by

$$
\phi_{+}(A x)=e^{\mp i \pi \Delta} \phi_{+}(x)
$$

under the antipodal map.

Given a bulk point $P$ contained inside some Poincaré patch, the global smearing function consists of three regions on the boundary. Region I is located to the past of the Poincaré patch, region II is contained within the Poincaré patch, and region III is to the future of the Poincaré patch. By applying a $\tau \rightarrow \tau+\pi$ antipodal map to region I, and a $\tau \rightarrow \tau-\pi$ antipodal map to region III, everything gets mapped inside the Poincare patch. Thus we can re-write the global smearing function as

$$
\begin{aligned}
& \phi(P)=\int d \tau d \Omega K_{\text {global }}(\tau, \Omega \mid P)\left(\phi_{0+}^{\text {global }}+\phi_{0-}^{\text {global }}\right) \\
& =\int_{\substack{\text { Poincare } \\
\text { patch }}}^{d \tau d \Omega} c_{d \Delta}|\sigma \cos \rho|^{\Delta-d}\left\{\begin{array}{ll}
e^{i \pi \Delta} \phi_{0+}^{\text {global }} & \text { in image of region I } \\
\phi_{0+}^{\text {global }} & \text { in region II } \\
e^{-i \pi \Delta} \phi_{0+}^{\text {global }} & \text { in image of region III }
\end{array}\right\}+\text { c.c. }
\end{aligned}
$$

where $c_{d \Delta}$ is the constant given in (20). We've used the fact that the integration measure is invariant under the antipodal map while $\sigma\left(x \mid x^{\prime}\right)=$ $-\sigma\left(A x \mid x^{\prime}\right)$. By regarding the phases as part of the smearing function rather than as part of the boundary field we have

$$
\phi(P)=\int_{\substack{\text { Poincare } \\
\text { patch }}} d \tau d \Omega c_{d \Delta}\left\{\begin{array}{c}
e^{i \pi \Delta} \\
1 \\
e^{-i \pi \Delta}
\end{array}\right\}|\sigma \cos \rho|^{\Delta-d} \phi_{0+}^{\text {global }}+\text { c.c. }
$$

Putting in the Jacobians to convert from global to Poincaré coordinates, namely

$$
\frac{d \tau d \Omega}{\cos ^{d} \rho}=\frac{d T d^{d-1} X}{Z^{d}}
$$

\footnotetext{
${ }^{5}$ It's defined on the AdS hyperboloid, so it's ambiguous whether $\tau$ is increased or decreased by $\pi$. We'll need both options below.
} 
and

$$
\cos ^{\Delta} \rho \phi_{0}^{\text {global }}=Z^{\Delta} \phi_{0}^{\text {Poincare }}
$$

this becomes

$$
\phi(P)=\int d T d^{d-1} X c_{d \Delta}\left\{\begin{array}{c}
e^{i \pi \Delta} \\
1 \\
e^{-i \pi \Delta}
\end{array}\right\}|\sigma Z|^{\Delta-d} \phi_{0+}^{\text {Poincare }}+\text { c.c. }
$$

Now consider the function

$$
f(T, X \mid P)=\lim _{Z \rightarrow 0}(\sigma(T, Z, X \mid P) Z)^{\Delta-d}
$$

defined with the prescription $T \rightarrow T-i \epsilon$. That is,

$$
\begin{aligned}
f(T) & =\left(\frac{1}{2 Z^{\prime}}\left(Z^{\prime 2}+\left|X-X^{\prime}\right|^{2}-\left(T-T^{\prime}-i \epsilon\right)^{2}\right)\right)^{\Delta-d} \\
& = \begin{cases}-e^{i \pi \Delta}|\sigma Z|^{\Delta-d} & \text { in image of region I } \\
|\sigma Z|^{\Delta-d} & \text { in region II } \\
-e^{-i \pi \Delta}|\sigma Z|^{\Delta-d} & \text { in image of region III }\end{cases}
\end{aligned}
$$

where we have used the fact that $d$ is odd. Since $f$ is analytic in the lower half complex $T$ plane its Fourier transform $f(\omega)=\int d T e^{i \omega T} f(T)$ vanishes for $\omega<0$, and hence $\int d T f(T) \phi_{0+}^{\text {Poincare }}=0$. We are therefore free to modify the Poincare smearing function by replacing $K_{+} \rightarrow K_{+}+c_{d \Delta} f$ in (34). This exactly cancels the smearing function at timelike separation, while giving a factor of two at spacelike separation, resulting in smearing function which is real. Thus in the end we obtain the Poincaré smearing function

$$
\begin{aligned}
& \phi(P)=\int d T d^{d-1} X K_{\text {Poincare }}(T, X \mid P) \phi_{0}^{\text {Poincare }}(T, X) \\
& \left.K_{\text {Poincare }}=2 c_{d \Delta} \lim _{Z \rightarrow 0}(\sigma(T, Z, X \mid P) Z)^{\Delta-d} \theta \text { (spacelike }\right) .
\end{aligned}
$$

Note that this smearing function grows at large spacelike separation. However a boundary field which is globally well-defined must fall off at large spacelike separation, $\phi_{0}^{\text {Poincare }} \sim \sigma^{-\Delta}$ as $X \rightarrow \infty$. So the convolution $\int K \phi_{0}$ is well-defined. 


\subsection{Odd AdS}

We now construct a Poincaré smearing function in odd-dimensional AdS, following the same procedure as in the last section: we start with the global result (33) and use the antipodal map to transform it into a Poincaré patch.

Applying the same logic as in the last section, we have

$$
\begin{aligned}
& \phi(P)=\int d \tau d \Omega K_{\text {global }}(\tau, \Omega \mid P)\left(\phi_{0+}^{\text {global }}+\phi_{0-}^{\text {global }}\right) \\
& =\int_{\substack{\text { Poincare } \\
\text { patch }}}^{d \tau d \Omega} a_{d \Delta}|\sigma \cos \rho|^{\Delta-d} \log |\sigma \cos \rho|\left\{\begin{array}{ll}
e^{i \pi \Delta} \phi_{0+}^{\text {global }} & \text { in image of region I } \\
\phi_{0+}^{\text {global }} & \text { in region II } \\
e^{-i \pi \Delta} \phi_{0+}^{\text {global }} & \text { in image of region III }
\end{array}\right\}+\text { c.c. }
\end{aligned}
$$

where $a_{d \Delta}$ is the constant given in (33). Again, regarding the phases as part of the smearing function rather than as part of the boundary field and transforming to Poincaré coordinates, we have

$$
\phi(P)=\int d T d^{d-1} X a_{d \Delta}\left\{\begin{array}{c}
e^{i \pi \Delta} \\
1 \\
e^{-i \pi \Delta}
\end{array}\right\}|\sigma Z|^{\Delta-d} \log |\sigma Z| \phi_{0+}^{\text {Poincare }}+\text { c.c. }
$$

This relies on the fact that, as shown in appendix B. we can replace $\log |\sigma \cos \rho|$ with $\log |\sigma Z|$ in the smearing function.

The phases in the smearing function can be absorbed into an $i \epsilon$ prescription. That is, we have

$$
\phi(P)=\int d T d^{d-1} X K_{+} \phi_{0+}^{\text {Poincare }}+\text { c.c. }
$$

where

$$
K_{+}=\left.a_{d \Delta}(\sigma Z)^{\Delta-d}\right|_{T \rightarrow T-i \epsilon} \log |\sigma Z| .
$$

A function analytic in the lower half $T$ plane gives vanishing result when integrated against $\phi_{0+}^{\text {Poincare }}$. So we can even take $K_{+}$to be given by the rather peculiar $i \epsilon$ prescription

$$
K_{+}=\left.\left.\frac{1}{2} a_{d \Delta}(\sigma Z)^{\Delta-d}\right|_{T \rightarrow T-i \epsilon} \log (\sigma Z)\right|_{T \rightarrow T+i \epsilon}
$$


Note that $K_{+}$isn't real in general, so we can't take $K_{+}=K_{-}=K$. Rather

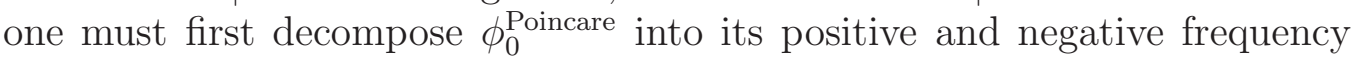
components before using these results. Also note that the smearing function is not restricted to spacelike separation. It is, however, AdS covariant.

It is not clear to us whether these peculiar features are fundamental to Poincaré smearing in odd dimensions, or can be overcome in some manner. However we would like to point out one exceptional case: if $\Delta$ is an integer then $K_{+}$can be taken to be real and we have

$$
\phi(P)=\int d T d^{d-1} X a_{d \Delta}(\sigma Z)^{\Delta-d} \log |\sigma Z| \phi_{0}^{\text {Poincare }}
$$

In appendix $\mathrm{C}$ we reproduce this result for $d=2$, starting from a Poincaré mode sum.

\section{Rindler smearing in $\mathrm{AdS}_{3}$}

We'll work in $\mathrm{AdS}_{3}$ in Rindler coordinates, with metric

$$
d s^{2}=-\frac{r^{2}-r_{+}^{2}}{R^{2}} d t^{2}+\frac{R^{2}}{r^{2}-r_{+}^{2}} d r^{2}+r^{2} d \phi^{2} .
$$

Here $-\infty<t, \phi<\infty$ and $r_{+}<r<\infty . \quad R$ is the AdS radius and $r_{+}$ is the radial position of the Rindler horizon. With the ansatz $\phi(t, r, \phi)=$ $e^{-i \omega t} e^{i k \phi} f_{\omega k}(r)$ a normalizeable solution to the scalar wave equation is [17, $[18]^{6}$

$$
f_{\omega k}(r)=r^{-\Delta}\left(\frac{r^{2}-r_{+}^{2}}{r^{2}}\right)^{-i \hat{\omega} / 2} F\left(\frac{\Delta-i \hat{\omega}-i \hat{k}}{2}, \frac{\Delta-i \hat{\omega}+i \hat{k}}{2}, \Delta, \frac{r_{+}^{2}}{r^{2}}\right)
$$

Here we define $\hat{\omega}=\omega R^{2} / r_{+}$and $\hat{k}=k R / r_{+}$. Perhaps despite appearances, the mode functions $f_{\omega k}$ are real and satisfy

$$
f_{\omega, k}=f_{\omega,-k}=f_{-\omega, k}=f_{-\omega,-k} .
$$

\footnotetext{
${ }^{6}$ The other solution to the differential equation grows like $r^{\Delta-d}$.
} 
Note that $-\infty<\omega, k<\infty$ so, unlike global and Poincaré, the Rindler modes involve a complete set of functions on the boundary [19]. This means we will have no freedom in choosing the Rindler smearing function. We therefore expect to find boundary operators which are as well-localized as possible.

The field has an expansion in Rindler modes

$$
\phi(t, r, \phi)=\int_{-\infty}^{\infty} d \omega \int_{-\infty}^{\infty} d k a_{\omega k} e^{-i \omega t} e^{i k \phi} f_{\omega k}(r)
$$

The Rindler boundary field is given by

$$
\phi_{0}(t, \phi)=\lim _{r \rightarrow \infty} r^{\Delta} \phi(t, r, \phi)=\int_{-\infty}^{\infty} d \omega \int_{-\infty}^{\infty} d k a_{\omega k} e^{-i \omega t} e^{i k \phi}
$$

so we can express

$$
a_{\omega k}=\frac{1}{4 \pi^{2}} \int d t d \phi e^{i \omega t} e^{-i k \phi} \phi_{0}(t, \phi)
$$

We can therefore represent the bulk field in terms of the boundary field as

$$
\phi(t, r, \phi)=\frac{1}{4 \pi^{2}} \int d \omega d k\left(\int d t^{\prime} d \phi^{\prime} e^{-i \omega\left(t-t^{\prime}\right)} e^{i k\left(\phi-\phi^{\prime}\right)} \phi_{0}\left(t^{\prime}, \phi^{\prime}\right)\right) f_{\omega k}(r) .
$$

If we were justified in changing the order of integration and doing the integrals over $\omega$ and $k$ first, we would have an expression for the Rindler smearing function which is just the Fourier transform of the mode functions.

$$
K\left(t^{\prime}, \phi^{\prime} \mid t, r, \phi\right) \stackrel{?}{=} \frac{1}{4 \pi^{2}} \int d \omega d k e^{-i \omega\left(t-t^{\prime}\right)} e^{i k\left(\phi-\phi^{\prime}\right)} f_{\omega k}(r)
$$

However the mode functions $f_{\omega k}$ diverge at large $k$, which means we can't simply change the order of integration; we need to proceed in a more careful way. We will find that a smearing function can be constructed by analytically continuing to imaginary values of the $\phi$ coordinate.

\subsection{Massless field in Rindler coordinates}

In this subsection we specialize to a massless field $(\Delta=2)$, and derive the smearing function in Rindler coordinates directly from (41) by a process of 
analytic continuation. It will be convenient to define the rescaled variables $\hat{t}=r_{+} t / R^{2}, \hat{\phi}=r_{+} \phi / R$ and $\hat{\omega}=w R^{2} / r_{+}, \hat{k}=k R / r_{+}$. We'll also use light-front coordinates

$$
\hat{\omega}^{ \pm}=\frac{1}{2}(\hat{\omega} \pm \hat{k}), \quad \hat{x}^{ \pm}=\hat{t} \pm \hat{\phi} .
$$

Let us start by rewriting (41) in the form

$$
\begin{aligned}
\phi(t, r, \phi)= & \frac{1}{4 \pi^{2}} \int d \omega d k \frac{1}{\cosh \left(\pi \hat{\omega}^{+} / 2\right) \cosh \left(\pi \hat{\omega}^{-} / 2\right)} f_{\omega k}(r) \\
& \left(\cosh \left(\pi \hat{\omega}^{+} / 2\right) \cosh \left(\pi \hat{\omega}^{-} / 2\right) \int d t^{\prime} d \phi^{\prime} e^{-i \omega\left(t-t^{\prime}\right)} e^{i k\left(\phi-\phi^{\prime}\right)} \phi_{0}\left(t^{\prime}, \phi^{\prime}\right)\right) .
\end{aligned}
$$

The point of breaking things up in this way is that it will lead to a welldefined kernel, since $f_{\omega k} \lesssim e^{\pi k / 2}$ as $k \rightarrow \infty$. We'll denote this modified kernel by $\tilde{K}$ below.

The hypergeometric function has an integral representation ([16], p. 78))

$F(a, b, c, z)=\frac{\Gamma(c)}{\Gamma(a) \Gamma(c-a)} \frac{\Gamma(c)}{\Gamma(b) \Gamma(c-b)} \int_{0}^{1} d s \int_{0}^{1} d t s^{a-1}(1-s)^{c-a-1} t^{b-1}(1-t)^{c-b-1}(1-s t z)^{-c}$.

Specializing to a massless field $(\Delta=2)$ this becomes

$$
\begin{aligned}
F\left(1-i \hat{\omega}^{+}, 1-i \hat{\omega}^{-}, 2, r_{+}^{2} / r^{2}\right)= & \frac{\sinh \pi \hat{\omega}^{+}}{\pi \hat{\omega}^{+}} \frac{\sinh \pi \hat{\omega}^{-}}{\pi \hat{\omega}^{-}} \int_{0}^{1} d s \int_{0}^{1} d t \\
& \left(\frac{s}{1-s}\right)^{-i \hat{\omega}^{+}}\left(\frac{t}{1-t}\right)^{-i \hat{\omega}^{-}}\left(1-s t r_{+}^{2} / r^{2}\right)^{-2}
\end{aligned}
$$

Using this to represent the mode functions in (43), the bulk field can be expressed as a convolution

$$
\phi\left(\hat{x}^{+}, \hat{x}^{-}, r\right)=\frac{1}{r^{\Delta}} \int d \hat{y}^{+} d \hat{y}^{-} \tilde{K}\left(\hat{x}^{+}-\hat{y}^{+}, \hat{x}^{-}-\hat{y}^{-}, r\right) \tilde{\phi}_{0}\left(\hat{y}^{+}, \hat{y}^{-}\right)
$$

where

$$
\begin{aligned}
& \tilde{K}= \int_{0}^{1} d s \int_{0}^{1} d t\left(1-s t r_{+}^{2} / r^{2}\right)^{-2} \int \frac{d \hat{\omega}^{+}}{2 \pi} \frac{d \hat{\omega}^{-}}{2 \pi} \frac{\sinh \left(\pi \hat{\omega}^{+} / 2\right)}{\pi \hat{\omega}^{+} / 2} \frac{\sinh \left(\pi \hat{\omega}^{-} / 2\right)}{\pi \hat{\omega}^{-} / 2} \\
& \exp \left[-i \hat{\omega}^{+}\left(\hat{x}^{-}-\hat{y}^{-}+\frac{1}{2} \log \left(1-r_{+}^{2} / r^{2}\right)-\log \frac{1-s}{s}\right]\right. \\
& \exp \left[-i \hat{\omega}^{-}\left(\hat{x}^{+}-\hat{y}^{+}+\frac{1}{2} \log \left(1-r_{+}^{2} / r^{2}\right)-\log \frac{1-t}{t}\right]\right.
\end{aligned}
$$


and where

$$
\begin{aligned}
\tilde{\phi}_{0}\left(\hat{y}^{+}, \hat{y}^{-}\right)= & \int \frac{d \hat{\omega}^{+}}{2 \pi} \frac{d \hat{\omega}^{-}}{2 \pi} \cosh \left(\pi \hat{\omega}^{+} / 2\right) \cosh \left(\pi \hat{\omega}^{-} / 2\right) \\
& \left(\int d \hat{y}^{\prime+} d \hat{y}^{\prime-} e^{-i \hat{\omega}^{+}\left(\hat{y}^{-}-\hat{y}^{-}\right)} e^{-i \hat{\omega}^{-}\left(\hat{y}^{+}-\hat{y}^{+}\right)} \phi_{0}\left(\hat{y}^{\prime+}, \hat{y}^{\prime-}\right)\right) .
\end{aligned}
$$

The modified boundary field $\tilde{\phi}_{0}$ can be defined by analytic continuation, as we have

$$
\begin{aligned}
\tilde{\phi}_{0}\left(\hat{y}^{+}, \hat{y}^{-}\right)= & \cosh \left(\frac{i \pi}{2} \frac{\partial}{\partial \hat{y}^{+}}\right) \cosh \left(\frac{i \pi}{2} \frac{\partial}{\partial \hat{y}^{-}}\right) \phi_{0}\left(\hat{y}^{+}, \hat{y}^{-}\right) \\
= & \frac{1}{4}\left(\phi_{0}\left(\hat{y}^{+}+\frac{i \pi}{2}-i \epsilon, \hat{y}^{-}+\frac{i \pi}{2}-i \epsilon\right)+\phi_{0}\left(\hat{y}^{+}+\frac{i \pi}{2}-i \epsilon, \hat{y}^{-}-\frac{i \pi}{2}+i \epsilon\right)\right. \\
& \left.\phi_{0}\left(\hat{y}^{+}-\frac{i \pi}{2}+i \epsilon, \hat{y}^{-}+\frac{i \pi}{2}-i \epsilon\right)+\phi_{0}\left(\hat{y}^{+}-\frac{i \pi}{2}+i \epsilon, \hat{y}^{-}-\frac{i \pi}{2}+i \epsilon\right)\right) .
\end{aligned}
$$

This assumes the boundary field is analytic in the strip $-\pi / 2<\operatorname{Im} \hat{y}^{+}, \operatorname{Im} \hat{y}^{-}<$ $\pi / 2$, which is true for boundary fields constructed from finite superpositions of the global boundary mode functions (5).

To compute the modified kernel $\tilde{K}$ it's convenient to first act with $\partial_{+} \partial_{-}$ to kill the $1 / \hat{\omega}^{+} \hat{\omega}^{-}$factor, and to define the $\sinh \left(\pi \hat{\omega}^{+} / 2\right) \sinh \left(\pi \hat{\omega}^{-} / 2\right)$ factor by analytic continuation. The $\hat{\omega}^{+}$and $\hat{\omega}^{-}$integrals then produce $\delta$-functions which can be used to do the integrals over $s$ and $t$. Finally, upon integrating with respect to $\hat{x}^{+}$and $\hat{x}^{-}$we find

$$
\begin{aligned}
& \tilde{K}\left(\hat{x}^{+}, \hat{x}^{-}\right)=\frac{4}{\pi^{2}} \frac{r^{2}}{r_{+}^{2}} \sinh \left(\frac{i \pi}{2} \frac{\partial}{\partial \hat{x}^{+}}\right) \sinh \left(\frac{i \pi}{2} \frac{\partial}{\partial \hat{x}^{-}}\right) \\
& \log \left[\left(1+\sqrt{1-\frac{r_{+}^{2}}{r^{2}}} e^{\hat{x}^{+}}\right)\left(1+\sqrt{1-\frac{r_{+}^{2}}{r^{2}}} e^{\hat{x}^{-}}\right)-\frac{r_{+}^{2}}{r^{2}}\right] \\
&=\frac{r^{2}}{\pi^{2} r_{+}^{2}} \log \frac{\left(1-r_{+}^{2} / r^{2}\right) \sinh ^{2} \hat{t}+\cosh ^{2} \hat{\phi}}{\left(1-r_{+}^{2} / r^{2}\right) \cosh ^{2} \hat{t}+\sinh ^{2} \hat{\phi}}
\end{aligned}
$$

Now let's go back to our expression for the bulk field (44). We'll break it up into two pieces, $\phi=\int \tilde{K} \tilde{\phi}_{0}=A+B$. The first piece $A$ includes the 


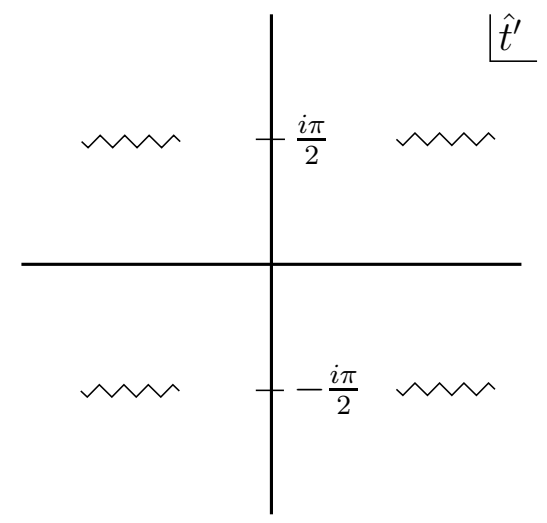

Figure 2: Branch cuts in the $\hat{t}^{\prime}$ plane are located at $\operatorname{Im} \hat{t}^{\prime}= \pm \pi / 2$.

terms in which the arguments of $\phi_{0}$ are shifted by $\hat{t} \rightarrow \hat{t} \pm i \pi / 2$. That is

$$
\begin{array}{r}
A=\frac{1}{2 \pi^{2} r_{+}^{2}} \int d \hat{t}^{\prime} d \hat{\phi}^{\prime} \log \frac{\left(1-r_{+}^{2} / r^{2}\right) \sinh ^{2}\left(\hat{t}-\hat{t}^{\prime}\right)+\cosh ^{2}\left(\hat{\phi}-\hat{\phi}^{\prime}\right)}{\left(1-r_{+}^{2} / r^{2}\right) \cosh ^{2}\left(\hat{t}-\hat{t}^{\prime}\right)+\sinh ^{2}\left(\hat{\phi}-\hat{\phi}^{\prime}\right)} \\
\left(\phi_{0}\left(\hat{t}^{\prime}+i \pi / 2-i \epsilon, \hat{\phi}^{\prime}\right)+\phi_{0}\left(\hat{t}^{\prime}-i \pi / 2+i \epsilon, \hat{\phi}^{\prime}\right)\right)
\end{array}
$$

The logarithm has branch cuts indicated in Fig. 2. By shifting the $\hat{t}^{\prime}$ contour of integration up or down by $i \pi / 2$ one can make the arguments of $\phi_{0}$ real. The imaginary parts of the logarithm just above and below the cuts cancel, while the real parts of the logarithm add to give

$$
A=\frac{1}{\pi^{2} r_{+}^{2}} \int_{-\infty}^{\infty} d \hat{t}^{\prime} \int_{-\infty}^{\infty} d \hat{\phi}^{\prime} \log \left|\frac{-\left(1-r_{+}^{2} / r^{2}\right) \cosh ^{2}\left(\hat{t}-\hat{t}^{\prime}\right)+\cosh ^{2}\left(\hat{\phi}-\hat{\phi}^{\prime}\right)}{-\left(1-r_{+}^{2} / r^{2}\right) \sinh ^{2}\left(\hat{t}-\hat{t}^{\prime}\right)+\sinh ^{2}\left(\hat{\phi}-\hat{\phi}^{\prime}\right)}\right| \phi_{0}\left(\hat{t}^{\prime}, \hat{\phi}^{\prime}\right)
$$

Now consider the contribution $B$ coming from terms where $\hat{\phi} \rightarrow \hat{\phi} \pm i \pi / 2$, namely

$$
\begin{array}{r}
B=\frac{1}{2 \pi^{2} r_{+}^{2}} \int d \hat{t}^{\prime} d \hat{\phi}^{\prime} \log \frac{\left(1-r_{+}^{2} / r^{2}\right) \sinh ^{2}\left(\hat{t}-\hat{t}^{\prime}\right)+\cosh ^{2}\left(\hat{\phi}-\hat{\phi}^{\prime}\right)}{\left(1-r_{+}^{2} / r^{2}\right) \cosh ^{2}\left(\hat{t}-\hat{t}^{\prime}\right)+\sinh ^{2}\left(\hat{\phi}-\hat{\phi}^{\prime}\right)} \\
\left(\phi_{0}\left(\hat{t}^{\prime}, \hat{\phi}^{\prime}+i \pi / 2-i \epsilon\right)+\phi_{0}\left(\hat{t}^{\prime}, \hat{\phi}^{\prime}-i \pi / 2+i \epsilon\right)\right)
\end{array}
$$

The branch cuts of the logarithm are shown in Fig. 3. One can push the $\hat{\phi}^{\prime}$ contour of integration up or down by $i \pi / 2$, however if $\cosh \left(\hat{t}-\hat{t}^{\prime}\right)<$ 

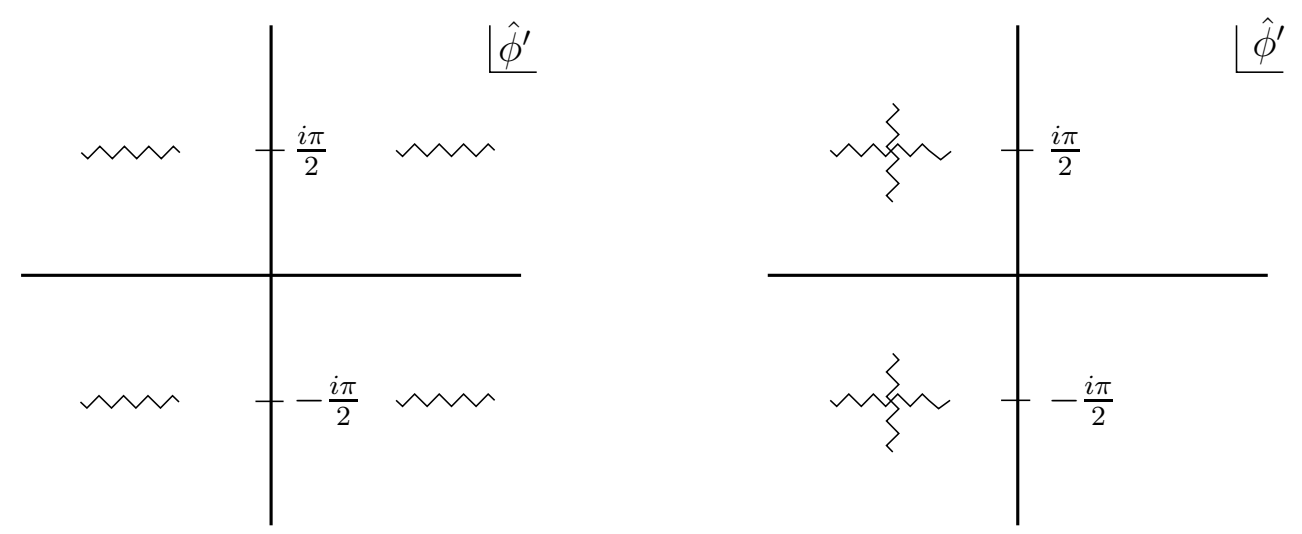

Figure 3: For $\cosh \left(\hat{t}-\hat{t}^{\prime}\right)>1 / \sqrt{1-r_{+}^{2} / r^{2}}$ the branch cuts in the $\hat{\phi}^{\prime}$ plane are at $\operatorname{Im} \hat{\phi}^{\prime}= \pm \pi / 2$ (left panel). When $\cosh \left(\hat{t}-\hat{t}^{\prime}\right)=1 / \sqrt{1-r_{+}^{2} / r^{2}}$ four of the branch points touch, and for $\cosh \left(\hat{t}-\hat{t}^{\prime}\right)<1 / \sqrt{1-r_{+}^{2} / r^{2}}$ the branch cuts are cross-shaped (right panel).

$1 / \sqrt{1-r_{+}^{2} / r^{2}}$ the contour will get wrapped around the vertical part of the cut. In fact, after shifting $\hat{\phi}^{\prime}$, the contribution from horizontal part of the $\hat{\phi}^{\prime}$ contour is

$B_{\text {horizontal }}=\frac{1}{\pi^{2} r_{+}^{2}} \int_{-\infty}^{\infty} d \hat{t}^{\prime} \int_{-\infty}^{\infty} d \hat{\phi}^{\prime} \log \left|\frac{-\left(1-r_{+}^{2} / r^{2}\right) \sinh ^{2}\left(\hat{t}-\hat{t}^{\prime}\right)+\sinh ^{2}\left(\hat{\phi}-\hat{\phi}^{\prime}\right)}{-\left(1-r_{+}^{2} / r^{2}\right) \cosh ^{2}\left(\hat{t}-\hat{t}^{\prime}\right)+\cosh ^{2}\left(\hat{\phi}-\hat{\phi}^{\prime}\right)}\right| \phi_{0}\left(\hat{t}^{\prime}, \hat{\phi}^{\prime}\right)$.

But this exactly cancels the contribution (477) from shifting $\hat{t}^{\prime}$ ! So the bulk field arises just from the vertical part of the contour, that is from the discontinuity of the logarithm across the cut. Setting $\hat{t}^{\prime}=\hat{t}+x, \hat{\phi}^{\prime}=\hat{\phi}+i y$ this leads to

$$
\begin{aligned}
& \phi(\hat{t}, r, \hat{\phi})=\frac{1}{2 \pi^{2} r_{+}^{2}} \int_{-x_{0}}^{x_{0}} d x {\left[\int_{-\pi / 2}^{-y_{0}} d y 2 \pi \phi_{0}(\hat{t}+x, \hat{\phi}+i y+i \pi / 2)\right.} \\
&\left.+\int_{y_{0}}^{\pi / 2} d y 2 \pi \phi_{0}(\hat{t}+x, \hat{\phi}+i y-i \pi / 2)\right]
\end{aligned}
$$

where $\cosh x_{0}=1 / \sqrt{1-r_{+}^{2} / r^{2}}$ and $\sin y_{0}=\sqrt{1-r_{+}^{2} / r^{2}} \cosh x$. Finally one can shift $y \rightarrow y \pm \pi / 2$ to obtain

$$
\phi(\hat{t}, r, \hat{\phi})=\frac{1}{\pi r_{+}^{2}} \int_{R} d x d y \phi_{0}(\hat{t}+x, \hat{\phi}+i y)
$$


where the region $R$ is defined by

$$
\begin{aligned}
& \cosh x<1 / \sqrt{1-r_{+}^{2} / r^{2}} \\
& \cos y>\sqrt{1-r_{+}^{2} / r^{2}} \cosh x .
\end{aligned}
$$

It is worth emphasizing that we have been forced to work on the complexified boundary. Had there been a well-defined Rindler smearing function, with support on the Rindler boundary at real values of the boundary coordinates, it would have been given by (42). Since that integral is divergent, no such smearing function can exist.

\subsection{Wick rotating to de Sitter space}

In this subsection we derive the Rindler smearing function for general conformal dimension. Having seen that we need to analytically continue the $\phi$ coordinate we begin by Wick rotating $\phi$ to $\tilde{\phi}=i \phi$. This gives a de Sitter geometry,

$$
d s^{2}=-\frac{r^{2}-r_{+}^{2}}{R^{2}} d t^{2}+\frac{R^{2}}{r^{2}-r_{+}^{2}} d r^{2}-r^{2} d \tilde{\phi}^{2} .
$$

Note that $-r$ plays the role of the time coordinate. To avoid a conical singularity at $r=0$ we must periodically identify $\tilde{\phi} \sim \tilde{\phi}+2 \pi R / r_{+}$. The de Sitter invariant distance function is

$$
\sigma=\frac{r r^{\prime}}{r_{+}^{2}}\left(\cos \left(\frac{r_{+}\left(\tilde{\phi}-\tilde{\phi}^{\prime}\right)}{R}\right)-\sqrt{1-\frac{r_{+}^{2}}{r^{2}}} \sqrt{1-\frac{r_{+}^{2}}{r^{\prime 2}}} \cosh \left(\frac{r_{+}\left(t-t^{\prime}\right)}{R^{2}}\right)\right)
$$

We consider a scalar field of mass $m$ in de Sitter space. For now we take $m^{2} R^{2}>1$, however later we will analytically continue $m^{2} \rightarrow-m^{2}$. The analytically continued mass can be identified with the mass of a field in AdS (note that the Wick rotation flips the signature of the metric).

The field at some bulk point can be written in terms of the retarded Greens function. de Sitter space has numerous inequivalent vacuum states, known as the alpha-vacua, which give rise to de Sitter invariant correlators. The retarded Greens function is independent of this choice of vacuum state. 
It coincides with the imaginary part of the commutator inside the past lightcone of the future point and vanishes outside this region. The field at some bulk point is therefore

$$
\phi(r, \tilde{\phi}, t)=\int d \tilde{\phi}^{\prime} d t^{\prime} \frac{r^{\prime}\left(r^{\prime 2}-r_{+}^{2}\right)}{R^{2}} G_{r e t}\left(r^{\prime}, \tilde{\phi}^{\prime}, t^{\prime} ; r, \tilde{\phi}, t\right) \overleftrightarrow{\partial_{r^{\prime}}} \phi\left(r^{\prime}, \tilde{\phi}^{\prime}, t^{\prime}\right)
$$

where the region of integration is over a spacelike surface of fixed $r^{\prime}$ inside the past light-cone of the bulk point. In the $r^{\prime} \rightarrow \infty$ limit this becomes the region $R$ introduced in (50), namely

$$
\begin{aligned}
& \sqrt{1-\frac{r_{+}^{2}}{r^{2}}} \cosh \frac{r_{+}\left(t-t^{\prime}\right)}{R^{2}}<1 \\
& \cos \frac{r_{+}\left(\tilde{\phi}-\tilde{\phi}^{\prime}\right)}{R}>\sqrt{1-\frac{r_{+}^{2}}{r^{2}}} \cosh \frac{r_{+}\left(t-t^{\prime}\right)}{R^{2}}
\end{aligned}
$$

As $r^{\prime} \rightarrow \infty$ (with other coordinates held fixed) the retarded Greens function takes the form [20]

$$
G_{r e t} \sim i\left(c(-\sigma-i \epsilon)^{-1+i \sqrt{m^{2} R^{2}-1}}+c^{*}(-\sigma-i \epsilon)^{-1-i \sqrt{m^{2} R^{2}-1}}-c . c .\right)
$$

where we take branch cuts along the positive real $\sigma$ axis and where

$$
c=\frac{\Gamma\left(2 i \sqrt{m^{2}-1}\right) \Gamma\left(1-i \sqrt{m^{2}-1}\right)}{2^{2-i \sqrt{m^{2}-1}} R \Gamma\left(\frac{1}{2}+i \sqrt{m^{2}-1}\right)} .
$$

The boundary field is defined as usual

$$
\phi_{0}(\tilde{\phi}, t)=\lim _{r \rightarrow \infty} r^{\Delta} \phi(r, \tilde{\phi}, t) .
$$

Choosing normalizable modes from the AdS viewpoint corresponds to taking only positive frequencies in the $-r$ direction, which have a $r^{-1-i \sqrt{m^{2}-1}} r$ dependence. 
Evaluating (51) as $r^{\prime} \rightarrow \infty$ we obtain the Rindler smearing function ${ }^{7}$

$$
\begin{aligned}
& \phi(r, \phi, t)=\frac{(\Delta-1) 2^{\Delta-2}}{\pi r_{+}^{2}} \int_{R} d x d y \lim _{r^{\prime} \rightarrow \infty}\left(\frac{\sigma}{r^{\prime}}\right)^{\Delta-2} \phi_{0}\left(\phi+i \frac{R y}{r_{+}}, t+\frac{R^{2} x}{r_{+}}\right) \\
& =\frac{(\Delta-1) 2^{\Delta-2}}{\pi r_{+}^{\Delta}} \int_{R} d x d y\left(\frac{r}{r_{+}}\left(\cos y-\sqrt{1-\frac{r_{+}^{2}}{r^{2}}} \cosh x\right)\right)^{\Delta-2} \\
& \times \phi_{0}\left(\phi+i \frac{R y}{r_{+}}, t+\frac{R^{2} x}{r_{+}}\right)
\end{aligned}
$$

In these expressions $\Delta=1+i \sqrt{m^{2}-1}$. However by analytically continuing $m^{2} \rightarrow-m^{2}$ we can take $\Delta$ to coincide with the conformal dimension in AdS. Since $\sigma>1$ in the domain of integration this analytic continuation is straightforward.

As a check on this result, note that for $\Delta=2$ we reproduce (49). As a further check we can examine the limit $r \rightarrow \infty$ where we should recover (53). In this limit the region of integration becomes very small so we can Taylor expand the smearing function, finding

$$
\begin{aligned}
\phi(r, \phi, t) & \sim \frac{(\Delta-1) 2^{\Delta-2}}{\pi r_{+}^{\Delta}} \phi_{0}(\phi, t) \int_{R} d x d y \frac{r^{\Delta-2}}{r_{+}^{\Delta-2}}\left(\frac{r_{+}^{2}}{2 r^{2}}-\frac{1}{2} x^{2}-\frac{1}{2} y^{2}\right)^{\Delta-2} \\
& =\frac{1}{r^{\Delta}} \phi_{0}(\phi, t)
\end{aligned}
$$

as expected.

In this section we have used the fact that $\phi_{0}$ is analytic on a strip in the complex $\phi$ plane centered on the real axis, which will be true for fields built out of any superposition of a finite number of global modes. The final result (54) is manifestly AdS covariant. We have checked that it is correct by setting $\phi_{0}$ equal to a plane wave $e^{-i \omega t} e^{i k \phi}$ and numerically evaluating the integrals over $x$ and $y$, finding values that agree with the corresponding bulk field $e^{-i \omega t} e^{i k \phi} f_{\omega k}(r)$.

\footnotetext{
${ }^{7}$ Here we use the identities $\sin \pi z=\frac{\pi}{\Gamma(z) \Gamma(1-z)}$ and $\frac{\Gamma(2 z)}{\Gamma(z) \Gamma(1 / 2+z)}=\frac{2^{2 z-1}}{\sqrt{\pi}}$.
} 


\section{Physical consequences}

\subsection{Bulk locality and UV/IR}

We have seen that one can define operators in the boundary theory that in the large $N$ limit describe a free local bulk field. This is not surprising, since this is the limit $l_{\text {Planck }} \rightarrow 0$ where classical supergravity is valid. The construction of these operators in terms of a mode sum makes it clear that by construction the two-point function of these CFT operators will reproduce the bulk two-point function. The two-point function is singular when bulk points are coincident or are lightlike separated. Since the smearing functions are finite and have compact support, it's easy to see that this singularity can only arise from UV singularities in the boundary theory. ${ }^{8}$ This means that regions inside the bulk are not related to a boundary theory with a conventional UV cutoff, so there is no UV/IR relationship in the sense of relating bulk IR and boundary UV cutoffs.

What about scale-radius duality? In global and Poincaré coordinates this duality is not manifest. As can be seen in figure 1 there may be a minimum smearing in the time direction which is related to radial position in the bulk, however the CFT operators are always completely smeared over the spatial directions of the boundary. In Rindler coordinates, on the other hand, we were able to reduce the smearing integral to a compact region of the complexified geometry, whose size shrinks to zero as the bulk point approaches the boundary. This makes scale-radius duality manifest. For example, a bulk point at radius $r$ gets smeared over a range of time $\delta t$ on the boundary given by

$$
\cosh \frac{r_{+} \delta t}{2 R^{2}}=1 / \sqrt{1-r_{+}^{2} / r^{2}}
$$

This is just the elapsed time between the point on the boundary which is lightlike to the future of the bulk point at the same value of $\phi$, and the point on the boundary which is lightlike to the past at the same $\phi$. The smearing is also over some finite region in imaginary $\phi$, as per (52).

\footnotetext{
${ }^{8}$ This was shown explicitly in [13].
} 


\subsection{Finite $N$ and the holographic bound}

We begin with a few remarks on the boundary commutator in the complexified geometry of section 4.2. The boundary Wightman two-point function is

$$
\left\langle\phi_{0}(t, \phi) \phi_{0}\left(\phi^{\prime}, t^{\prime}\right)\right\rangle \sim\left(\cosh \frac{r_{+} \delta \phi}{R}-\cosh \left(\frac{r_{+} \delta t}{R^{2}}-i \epsilon\right)\right)^{-2 \Delta} .
$$

In the large $N$ limit, with free bulk fields, the commutator is a c-number which vanishes whenever the $i \epsilon$ term can be neglected. We are interested in real $t$ and complex $\phi$. So at large $N$ the commutator is non-zero in only two situations:

1. $\delta \phi$ real and $\delta t>R \delta \phi$ (the usual case of timelike separation),

2. $\delta \phi$ purely imaginary and $\delta t$ arbitrary,

while for generic complex $\delta \phi$ the commutator vanishes. What happens at finite $N$ ? Since the vacuum two-point function is determined by conformal invariance, (57) is true even at finite $N$. However the commutator is an operator rather than a c-number, so we cannot conclude that the commutator (rather than its vacuum expectation value) vanishes. Still, it seems reasonable to assume that up to $1 / N$ corrections to the size of the regions, the commutator will be non-zero only if condition 1 or 2 is satisfied.

At infinite $N$ the smeared operators we have constructed commute when the bulk points are spacelike separated. This works, even when the smeared operators overlap on the boundary, because the commutator of the boundary operators is a c-number rather than an operator. At finite $N$ this picture must change in an interesting way if a holographic description is to be maintained. Commutators of boundary operators become operators rather than c-numbers, which destroys the delicate balance that enabled two operators smeared over regions timelike to each other to commute. While the generalization of bulk field operators to finite $N$ is very difficult [6], we nevertheless get tight constraints from the $N \rightarrow \infty$ limit.

We argue that the only generic way for two smeared operators to commute at finite $N$ is by smearing over disjoint "spacelike" (commuting) regions on the boundary. This motivates representing local bulk operators using a form 
of the smearing function with minimal spread on the boundary, in the hope that such boundary operators provide the "most local" definition of bulk operators at finite $N$. We proceeded to this goal in a series of steps, first reducing from smearing operators over the entire boundary, to only smearing over points spacelike separated from the bulk point, and finally to smearing over a compact region of the complexified geometry. ${ }^{9}$ This prepares us to count the number of independent commuting bulk operators inside a given volume.

Consider two local bulk operators at the same values of $r$ and $t$ but different $\phi$. Up to $1 / N$ corrections to the actual size of the region, these will correspond to boundary operators smeared in the $t$ and imaginary $\phi$ directions according to (52). It therefore is reasonable to assume that even at finite $N$ these operators will commute if the $\phi$ separation is sufficiently large that the boundary commutator always vanishes. This requires

$$
\cosh \frac{r_{+} \delta \phi}{2 R}>1 / \sqrt{1-r_{+}^{2} / r^{2}}
$$

Let us work at large $r$. Then we expect bulk operators separated by $\delta \phi=$ $2 R / r$ to commute at finite $N$. Consider the set of such operators at fixed $r$ and $t$. Operators at smaller values of $r$ and the same $t$ will be smeared over a larger time interval on the boundary, so will not trivially commute with this set. Then the number of trivially commuting operators that can be localized to a radius $\leq r$, per radian along the boundary, per independent CFT degree of freedom is $r / 2 R$. Heuristically the number of CFT degrees of freedom is given by the central charge, so the maximum number of commuting operators per radian is of order

$$
\mathrm{cr} / 2 R \text {. }
$$

This result is consistent with expectations from holography, and gives a nice picture of how the number of commuting degrees of freedom is drastically reduced. This also makes it clear where canonical quantization of gravity fails: the degrees of freedom on a Cauchy hypersurface do not commute. Note that if $\phi$ is periodically identified to give a BTZ black hole, $\phi \sim \phi+2 \pi$, then this counting breaks down when $2 R / r \approx 2 \pi$ and the operator is smeared over the entire boundary. Such a breakdown is expected, since a HawkingPage transition occurs for a black hole of radius $r \sim R[21$.

\footnotetext{
${ }^{9}$ Although we explicitly performed this last step only for three dimensions in Rindler coordinates, it is straightforward to generalize to arbitrary dimensions and other coordinates systems.
} 


\section{Acknowledgements}

We thank Lenny Susskind and Shubho Roy for valuable discussions. The work of AH and DK is supported by US Department of Energy grant DEFG02-92ER40699. GL is supported in part by a grant from the Israeli science foundation number 568/05. The research of DL is supported in part by DOE grant DE-FG02-91ER40688-Task A.

\section{A Even AdS: global Greens function}

In this section we show how to reproduce our global smearing function in even-dimensional AdS starting from a spacelike Greens function. To construct such a Greens function we first find the general (singular) AdSinvariant solution to the homogeneous wave equation in Euclidean space. The solution involves two arbitrary constants. We fix one constant by requiring that the solution is in fact a Greens function with a properly-normalized delta-function source at the origin. We fix the other constant by requiring that, upon analytically continuing to Lorentzian AdS, the Greens function is non-zero only at spacelike separation.

The AdS-invariant distance (3) is defined for Lorentzian AdS. However by Wick rotating $\tau=-i \tau_{E}$ one can also use $\sigma$ as an invariant distance function on Euclidean AdS; continuing back to Lorentzian signature corresponds to the prescription $\sigma \rightarrow \sigma+i \epsilon$. For AdS-invariant fields ${ }^{10}$ the wave equation $\left(\square-m^{2}\right) \phi=0$ reduces to

$$
\left(\sigma^{2}-1\right) \phi^{\prime \prime}+(d+1) \sigma \phi^{\prime}-\Delta(\Delta-d) \phi=0 .
$$

The general solution is

$$
\phi(\sigma)=c_{1}\left(\sigma^{2}-1\right)^{-\mu / 2} P_{\nu}^{\mu}(\sigma)+c_{2}\left(\sigma^{2}-1\right)^{-\mu / 2} Q_{\nu}^{\mu}(\sigma)
$$

where $P_{\nu}^{\mu}, Q_{\nu}^{\mu}$ are associated Legendre functions with $\mu=\frac{D-2}{2}, \nu=\Delta-\frac{D}{2}$. In even AdS note that $\mu$ is a non-negative integer, in which case as $\sigma \rightarrow 1$

\footnotetext{
${ }^{10}$ Where we use the notation $\phi(x)=\phi\left(\sigma\left(x \mid x^{\prime}\right)\right)$.
} 
the Legendre functions have the asymptotic behavior [16]

$P_{\nu}^{\mu}(\sigma) \sim \frac{2^{-\mu / 2} \Gamma(\nu+\mu+1)(\sigma-1)^{\mu / 2}}{\mu ! \Gamma(\nu-\mu+1)}, \quad Q_{\nu}^{\mu}(\sigma) \sim 2^{\mu / 2-1} \Gamma(\mu) e^{i \pi \mu}(\sigma-1)^{-\mu / 2}$.

A Euclidean Greens function should have the short-distance behavior

$$
G_{E}(r) \sim-\frac{1}{(D-2) \operatorname{vol}\left(S^{d}\right) r^{D-2}} \quad \text { as } r \rightarrow 0
$$

where $r$ is a Euclidean radial coordinate and $\operatorname{vol}\left(S^{d}\right)=2 \pi^{D / 2} / \Gamma(D / 2)$. At short distances $\sigma \approx 1+r^{2} / 2 R^{2}$. So $\phi(\sigma)$ will be a Euclidean Greens function with a properly normalized (unit-strength) delta-function source at the origin provided

$$
c_{1}=\text { arbitrary }, \quad c_{2}=\frac{(-1)^{\mu+1}}{2^{\mu-1}(D-2) \operatorname{vol}\left(S^{d}\right) \Gamma(\mu) R^{D-2}} .
$$

Wick rotating back to Lorentzian AdS we set $G_{M}(\sigma)=i \phi(\sigma+i \epsilon)$ so that

$$
\left(\square-m^{2}\right) G_{M}=\frac{1}{\sqrt{-g}} \delta^{D}(x) .
$$

$G_{M}$ has the same short-distance behavior as the standard Feynman Greens function, although we have not yet fixed its large-distance behavior (which depends on $c_{1}$ ).

We choose $c_{1}$ to make the Greens function vanish at timelike separation. With a $\sigma \rightarrow \sigma+i \epsilon$ prescription the analytic continuation into the so-called "cut" region $-1<\sigma<1$ is [16]

$G_{M}(\sigma)=i c_{1}(-1)^{\mu}\left(1-\sigma^{2}\right)^{-\mu / 2} \hat{P}_{\nu}^{\mu}(\sigma)+i c_{2}(-1)^{\mu}\left(1-\sigma^{2}\right)^{-\mu / 2}\left(\hat{Q}_{\nu}^{\mu}(\sigma)-\frac{i \pi}{2} \hat{P}_{\nu}^{\mu}(\sigma)\right)$

where $\hat{P}, \hat{Q}$ are variants of the associated Legendre functions (denoted with upright $P$ 's and $Q$ 's in [16]) which are real for $-1<\sigma<1$. Since (65) is a real equation we only need to keep the real part of $G_{M}$. On the interval $-1<\sigma<1$ this is given by

$$
\operatorname{Re} G_{M}(\sigma)=\left(\operatorname{Re}\left(i c_{1}\right)+\frac{\pi c_{2}}{2}\right)(-1)^{\mu}\left(1-\sigma^{2}\right)^{-\mu / 2} \hat{P}_{\nu}^{\mu}(\sigma) .
$$


Note that $\operatorname{Re} G_{M}$ vanishes for $-1<\sigma<1$ provided $c_{1}=i \pi c_{2} / 2$. With this choice we can construct a new Greens function $G$ which is non-zero only at spacelike separation: ${ }^{11}$

$$
\begin{aligned}
G\left(x \mid x^{\prime}\right) & \equiv \begin{cases}\operatorname{Re} G_{M}\left(x \mid x^{\prime}\right) & \text { at spacelike separation } \\
0 & \text { otherwise }\end{cases} \\
& =-\frac{\pi c_{2}}{2}\left(\sigma^{2}-1\right)^{-\mu / 2} P_{\nu}^{\mu}(\sigma) \theta \text { (spacelike) }
\end{aligned}
$$

We can plug this into Green's identity

$$
\phi\left(x^{\prime}\right)=\left.\int d \tau d \Omega \sqrt{g_{\Omega}} \frac{R^{D-2}}{\cos ^{D-2} \rho}\left(\phi \partial_{\rho} G-G \partial_{\rho} \phi\right)\right|_{\rho \rightarrow \pi / 2}
$$

to obtain the corresponding smearing function. Noting the asymptotic behavior

$$
P_{\nu}^{\mu}(\sigma) \sim \frac{2^{\nu} \Gamma(\nu+1 / 2) \sigma^{\nu}}{\sqrt{\pi} \Gamma(\nu-\mu+1)} \quad \text { as } \sigma \rightarrow \infty
$$

we have

$$
\phi\left(x^{\prime}\right)=\int d \tau d \Omega \sqrt{g_{\Omega}} K\left(x \mid x^{\prime}\right) \phi_{0}(x)
$$

where the smearing function is

$K\left(x \mid x^{\prime}\right)=\frac{(-1)^{(D-2) / 2} 2^{\Delta-D} \Gamma\left(\Delta-\frac{d}{2}+1\right)}{\pi^{d / 2} \Gamma(\Delta-d+1)} \lim _{\rho \rightarrow \pi / 2}\left(\sigma\left(x \mid x^{\prime}\right) \cos \rho\right)^{\Delta-d} \theta$ (spacelike).

This agrees with the result (20) obtained from a global mode sum in even AdS.

\section{B AdS covariance in odd dimensions}

To show that the smearing function is AdS covariant in odd dimensions we must show that

$$
\int d \tau d \Omega \lim _{\rho_{0} \rightarrow \pi / 2}\left(\sigma \cos \rho_{0}\right)^{\Delta-d} \ln J \phi_{0}^{\text {global }}=0
$$

\footnotetext{
${ }^{11} G_{M}\left(x \mid x^{\prime}\right)$ also has delta function sources at all the $2 \pi$ images of the point $x^{\prime}$. By restricting $G$ to points that are spacelike separated from $x^{\prime}$ we keep only a single source.
} 
where $J$ is the Jacobian on the boundary induced by an AdS transformation of the bulk. Consider an AdS isometry which takes a point in the bulk to $\rho=0$. To compute the corresponding Jacobian introduce the embedding coordinates

$$
\begin{aligned}
Y_{0} & =R \sec \rho \cos \tau=\frac{1}{2 Z}\left(R^{2}+r^{2}+Z^{2}-T^{2}\right) \\
Y_{1} & =R \sec \rho \sin \tau=R \frac{T}{Z} \\
X_{0} & =R \tan \rho w_{0}=\frac{1}{2 Z}\left(R^{2}-r^{2}-Z^{2}+T^{2}\right) \\
\vec{X} & =R \tan \rho \vec{w}=R \frac{\vec{X}}{Z}
\end{aligned}
$$

where $w_{0}, \vec{w}$ are coordinates on a $(D-2)$-sphere. AdS transformations are given by rotations and boosts in these coordinates. The center of AdS, at $\rho=0$, is given in the embedding coordinates by $X_{0}=\vec{X}=0$. Given an arbitrary point in the bulk we can set $\vec{X}=Y_{1}=0$ by performing angular rotations and $\tau$ translations in global coordinates, for which the Jacobian is unity. We then boost in the $X_{0}-Y_{0}$ plane, resulting in a change of coordinates

$$
\begin{aligned}
X_{0}^{\prime} & =-\sinh \alpha Y_{0}+\cosh \alpha X_{0} \\
Y_{0}^{\prime} & =\cosh \alpha Y_{0}-\sinh \alpha X_{0} .
\end{aligned}
$$

Since we want $X_{0}^{\prime}=0$, this determines the parameter $\tanh \alpha=X_{0} / Y_{0}$. Now, turning our attention to the boundary, the Jacobian of the transformation is

$$
\begin{aligned}
J & =\lim _{\rho_{0} \rightarrow \pi / 2} \cos \rho_{0} / \cos \rho_{0}^{\prime} \\
\Rightarrow \quad J^{2} & =\frac{Y_{0}^{\prime 2}+Y_{1}^{\prime 2}}{Y_{0}^{2}+Y_{1}^{2}} \\
& =\frac{\left(\cosh \alpha Y_{0}-\sinh \alpha X_{0}\right)^{2}+Y_{1}^{2}}{Y_{0}^{2}+Y_{1}^{2}} .
\end{aligned}
$$

The strategy is to show that the integrand of (73) is analytic in the lower half complex plane, making the contour integral vanish. We find it convenient to perform the calculation in a variation on lightfront Poincaré coordinates, given by $X^{ \pm}=T \pm r$ with $r=|\vec{X}|$. However we have to be careful regarding the domain of integration of $X^{ \pm}$, since the spatial distance $r \geq 0$. If we 
restrict the domain of the angular coordinates on the boundary to cover only half the $d-2$ sphere, and instead allow $-\infty<r<\infty$, then $X^{ \pm}$has the full range of integration. One note, however. The integration measure in these new coordinates is proportional to $|r|^{d-2}$. In odd dimensional AdS, where $d$ is even, the measure factor is analytic. This would not be true in even dimensional AdS.

As in section 3.2 we project all boundary points into one Poincaré patch. Referring to (74) - (177) we want to evaluate the integral

$$
\begin{aligned}
I & =\left.\int d^{d} x\left(\sigma Z_{0}\right)^{\Delta-d}\right|_{T \rightarrow T-i \epsilon} \ln \left(\frac{\cos \rho_{0}^{\prime}}{\cos \rho_{0}}\right) \phi_{0+}^{\text {Poincare }}+\text { c.c. } \\
& =\left.\frac{1}{2} \int d^{d} x\left(\sigma Z_{0}\right)^{\Delta-d}\right|_{T \rightarrow T-i \epsilon}\left\{\ln \left[\left(R^{2}+r^{2}-T^{2}\right)^{2}+4 R^{2} T^{2}\right]\right. \\
& \left.-\ln \left[\left(\cosh \alpha\left(R^{2}+r^{2}-T^{2}\right)-\sinh \alpha\left(R^{2}-r^{2}+T^{2}\right)\right)^{2}+4 R^{2} T^{2}\right]\right\} \phi_{0+}^{\text {Poincare }}+\text { c.c. } \\
& =\left.\frac{1}{2} \int d^{d} x\left(\sigma Z_{0}\right)^{\Delta-d}\right|_{T \rightarrow T-i \epsilon}\left\{\ln \left(R^{2}+\left(X^{+}\right)^{2}\right)+\ln \left(R^{2}+\left(X^{-}\right)^{2}\right)\right. \\
& \left.-\ln \left(e^{\alpha} R^{2}+e^{-\alpha}\left(X^{+}\right)^{2}\right)-\ln \left(e^{\alpha} R^{2}+e^{-\alpha}\left(X^{-}\right)^{2}\right)\right\} \phi_{0+}^{\text {Poincare }}+\text { c.c. }
\end{aligned}
$$

We now show that the integrand is analytic in the lower half plane of one of the lightfront coordinates. With our $T \rightarrow T-i \epsilon$ prescription the branch points of $\left(\sigma Z_{0}\right)^{\Delta-d}$ are in the upper half complex plane of both $X^{ \pm}$. Each log term is independent of either $X^{+}$or $X^{-}$, and so is trivially analytic in that coordinate. Finally the boundary field contains terms like $\left.e^{-i\left(\omega^{+} X^{-}+\omega^{-} X^{+}\right.}\right)$ where $\omega^{ \pm}=\omega \pm|k| \cos \theta, \theta$ being the angle between $\vec{X}$ and the momentum $\vec{k}$. Note that $\omega^{ \pm} \geq 0$, due to the fact that $\omega \geq|k|$, so the boundary field is analytic in the lower half complex plane of both $X^{ \pm}$. This shows that the contour integral over one of the lightfront coordinates is zero, so $I$ vanishes and the smearing function is AdS covariant.

The same procedure can be used when converting the smearing function from global coordinates to Poincaré, showing that

$$
\int d^{d} x\left(\sigma Z_{0}\right)^{\Delta-d} \ln \left(\frac{Z_{0}}{\cos \rho_{0}}\right) \phi_{0}^{\text {Poincare }}=0 .
$$




\section{Odd AdS: Poincaré mode sum}

In Poincaré coordinates it is possible to construct a smearing function by directly evaluating the Poincaré mode sum. Bena did this in $\mathrm{AdS}_{5}$ [10; here we'll do the analogous calculation in $\mathrm{AdS}_{3}$.

In Poincaré coordinates the mode expansion of a real scalar field is

$$
\phi(T, X, Z)=\int_{\omega>|k|} d \omega d k a_{\omega k} e^{-i \omega T} e^{i k X} Z J_{\nu}\left(\sqrt{\omega^{2}-k^{2}} Z\right)+\text { c.c. }
$$

where $J_{\nu}$ is a Bessel function of order $\nu=\Delta-1$. The Poincaré boundary field is

$$
\begin{aligned}
\phi_{0}(T, X) & =\lim _{Z \rightarrow 0} \frac{1}{Z^{\Delta}} \phi(T, X, Z) \\
& =\frac{1}{2^{\Delta-1} \Gamma(\Delta)} \int_{\omega>|k|} d \omega d k a_{\omega k}\left(\omega^{2}-k^{2}\right)^{(\Delta-1) / 2} e^{-i \omega T} e^{i k X} .
\end{aligned}
$$

Thus we can express the bulk field in terms of the boundary field,

$$
\phi(T, X, Z)=\int d T^{\prime} d X^{\prime} K\left(T^{\prime}, X^{\prime} \mid T, X, Z\right) \phi_{0}\left(T^{\prime}, X^{\prime}\right)
$$

where the smearing function is

$$
\begin{aligned}
K\left(T^{\prime}, X^{\prime} \mid T, X, Z\right)= & \frac{2^{\Delta-3} \Gamma(\Delta) Z}{\pi^{2}} \int_{\omega>|k|} d \omega d k e^{-i \omega\left(T-T^{\prime}\right)} e^{i k\left(X-X^{\prime}\right)} \\
& \frac{1}{\left(\omega^{2}-k^{2}\right)^{(\Delta-1) / 2}} J_{\nu}\left(\sqrt{\omega^{2}-k^{2}} Z\right)+\text { c.c. }
\end{aligned}
$$

To keep the integral convergent we should give $T^{\prime}$ a positive imaginary part.

It's straightforward to evaluate the positive-frequency part of the smearing function. It suffices to set $T=X=X^{\prime}=0$ and consider

$K_{+}\left(T^{\prime}, 0 \mid 0,0, Z\right)=\frac{2^{\Delta-3} \Gamma(\Delta) Z}{\pi^{2}} \int_{\omega>|k|} d \omega d k e^{i \omega T^{\prime}} \frac{1}{\left(\omega^{2}-k^{2}\right)^{(\Delta-1) / 2}} J_{\nu}\left(\sqrt{\omega^{2}-k^{2}} Z\right)$

Setting

$$
\omega^{+}=\frac{1}{2}(\omega+k)=r e^{\xi} \quad \omega^{-}=\frac{1}{2}(\omega-k)=r e^{-\xi}
$$


we have

$$
\begin{aligned}
K_{+} & =\frac{2^{\Delta-1} \Gamma(\Delta) Z}{\pi^{2}} \int_{0}^{\infty} r d r \int_{-\infty}^{\infty} d \xi e^{i 2 r T^{\prime} \cosh \xi} \frac{1}{(2 r)^{\nu}} J_{\nu}(2 r Z) \\
& =\frac{2^{\Delta-2} \Gamma(\Delta) Z}{\pi^{2}} \int_{0}^{\infty} d r \frac{1}{r^{\nu-1}} K_{0}\left(-i r T^{\prime}\right) J_{\nu}(r Z) \\
& =-\frac{Z^{\Delta}}{2 \pi^{2} T^{\prime 2}} F\left(1,1, \Delta, Z^{2} / T^{\prime 2}\right) .
\end{aligned}
$$

The Lorentz-invariant generalization is

$$
K_{+}\left(T^{\prime}, X^{\prime} \mid 0,0, Z\right)=-\frac{1}{2 \pi^{2}} \frac{Z^{\Delta}}{T^{\prime 2}-X^{\prime 2}} F\left(1,1 ; \Delta ; \frac{Z^{2}}{T^{\prime 2}-X^{\prime 2}}\right)
$$

where again the singularities are to be handled with a $T^{\prime} \rightarrow T^{\prime}+i \epsilon$ prescription. Since $K_{+}$is constructed from positive-frequency modes its complex conjugate $K_{-}$only involves negative frequency modes. Then $\int K_{-} \phi_{0+}$ vanishes, and we can take the full smearing function to be given by $K=K_{+}+K_{-}$.

Note that the smearing function we have constructed has support on the entire boundary of the Poincaré patch. Also it can be applied directly to the boundary field $\phi_{0}$; unlike the smearing function constructed in section 3.2 one does not have to decompose $\phi_{0}$ into its positive and negative frequency components. It does have one drawback, however: the smearing function we have constructed is not AdS-covariant.

One might ask how the Poincaré mode sum is related to the covariant results obtained in section 3.2 . This is easiest to understand when $\Delta$ is an integer, in which case one has

$$
\begin{aligned}
\xi F(1,1, \Delta, \xi) & =-(\Delta-1)\left(1-\frac{1}{\xi}\right)^{\Delta-2} \log (1-\xi) \\
& +(\text { polynomial of degree } \Delta-3 \text { in } 1 / \xi)
\end{aligned}
$$

Applying this to (83) gives

$$
\begin{gathered}
K_{+}=\frac{\Delta-1}{2 \pi^{2}}\left(\frac{-T^{\prime 2}+X^{\prime 2}+Z^{2}}{Z}\right)^{\Delta-2} \log \frac{T^{\prime 2}-X^{\prime 2}-Z^{2}}{T^{\prime 2}-X^{\prime 2}} \\
+\left(\text { polynomial in } \frac{T^{\prime 2}-X^{\prime 2}}{Z^{2}}\right)
\end{gathered}
$$


We can drop the polynomial, since it vanishes when integrated against $\phi_{0+}$ (close the contour in the lower half $T^{\prime}$ plane). Also we can write

$$
\begin{aligned}
K_{+}=\frac{\Delta-1}{2 \pi^{2}} & \left(\frac{-T^{\prime 2}+X^{\prime 2}+Z^{2}}{Z}\right)^{\Delta-2} \log \frac{T^{\prime 2}-X^{\prime 2}-Z^{2}}{2 Z} \\
+ & (\text { polynomial }) \cdot \log \frac{\left(T^{\prime}+X^{\prime}\right)\left(T^{\prime}-X^{\prime}\right)}{2 Z}
\end{aligned}
$$

The second line vanishes when integrated against $\phi_{0+}$. To see this recall that the Poincaré mode expansion (79) only involves modes with $\omega>|k|$, and close the integration contour in the lower half of the $T^{\prime}+X^{\prime}$ or $T^{\prime}-X^{\prime}$ plane as appropriate. Then we are left with

$$
\begin{aligned}
K_{+} & =\frac{\Delta-1}{2 \pi^{2}}\left(\frac{-T^{\prime 2}+X^{\prime 2}+Z^{2}}{Z}\right)^{\Delta-2} \log \frac{T^{\prime 2}-X^{\prime 2}-Z^{2}}{2 Z} \\
& =\frac{(\Delta-1) 2^{\Delta-2}}{2 \pi^{2}} \lim _{Z^{\prime} \rightarrow 0}\left(\sigma Z^{\prime}\right)^{\Delta-2} \log \left(\sigma Z^{\prime}\right) .
\end{aligned}
$$

We can replace $K_{+} \rightarrow K_{+}+$c.c., since the complex conjugate drops out when integrated against $\phi_{0+}$. This leaves

$$
K=K_{+}=K_{-}=\frac{(\Delta-1) 2^{\Delta-2}}{\pi^{2}} \lim _{Z^{\prime} \rightarrow 0}\left(\sigma Z^{\prime}\right)^{\Delta-2} \log \left|\sigma Z^{\prime}\right|
$$

in agreement with (40) for $d=2$.

\section{References}

[1] J. M. Maldacena, "The large N limit of superconformal field theories and supergravity," Adv. Theor. Math. Phys. 2 (1998) 231-252, hep-th/9711200

[2] S. S. Gubser, I. R. Klebanov, and A. M. Polyakov, "Gauge theory correlators from non-critical string theory," Phys. Lett. B428 (1998) 105-114, hep-th/9802109.

[3] E. Witten, "Anti-de Sitter space and holography," Adv. Theor. Math. Phys. 2 (1998) 253-291, hep-th/9802150. 
[4] O. Aharony, S. S. Gubser, J. M. Maldacena, H. Ooguri, and Y. Oz, "Large N field theories, string theory and gravity," Phys. Rept. 323 (2000) 183-386, hep-th/9905111.

[5] V. Balasubramanian, P. Kraus, and A. E. Lawrence, "Bulk vs. boundary dynamics in anti-de Sitter spacetime," Phys. Rev. D59 (1999) 046003, hep-th/9805171.

[6] T. Banks, M. R. Douglas, G. T. Horowitz, and E. J. Martinec, "AdS dynamics from conformal field theory," hep-th/9808016.

[7] V. Balasubramanian, P. Kraus, A. E. Lawrence, and S. P. Trivedi, "Holographic probes of anti-de Sitter space-times," Phys. Rev. D59 (1999) 104021, hep-th/9808017.

[8] I. R. Klebanov and E. Witten, "AdS/CFT correspondence and symmetry breaking," Nucl. Phys. B556 (1999) 89-114, hep-th/9905104.

[9] V. Balasubramanian, S. B. Giddings, and A. E. Lawrence, "What do CFTs tell us about anti-de Sitter spacetimes?," JHEP 03 (1999) 001, hep-th/9902052

[10] I. Bena, "On the construction of local fields in the bulk of AdS(5) and other spaces," Phys. Rev. D62 (2000) 066007, hep-th/9905186.

[11] K. H. Rehren, "Algebraic holography," Annales Henri Poincare $\mathbf{1}$ (2000) 607-623, hep-th/9905179.

[12] K.-H. Rehren, "Local quantum observables in the anti-de Sitter conformal QFT correspondence," Phys. Lett. B493 (2000) 383-388, hep-th/0003120

[13] A. Hamilton, D. Kabat, G. Lifschytz, and D. A. Lowe, "Local bulk operators in AdS/CFT: A boundary view of horizons and locality," Phys. Rev. D73 (2006) 086003, hep-th/0506118.

[14] L. Susskind and E. Witten, "The holographic bound in anti-de Sitter space," hep-th/9805114.

[15] A. Peet and J. Polchinski, "UV/IR relations in AdS dynamics," Phys Rev. D59 (1999) 065011, hep-th/9809022. 
[16] A. Erdélyi, Higher transcendental functions, vol. I. McGraw-Hill, 1953.

[17] I. Ichinose and Y. Satoh, "Entropies of scalar fields on three-dimensional black holes," Nucl. Phys. B. 447 (1995) 340, hep-th/9412144.

[18] E. Keski-Vakkuri, "Bulk and boundary dynamics in BTZ black holes," Phys. rev. D59 (1999) 104001, hep-th/9808037.

[19] W. G. Unruh, "Notes on black hole evaporation," Phys. Rev. D14 (1976) 870.

[20] R. Bousso, A. Maloney, and A. Strominger, "Conformal vacua and entropy in de Sitter space," Phys. Rev. D65 (2002) 104039, hep-th/0112218.

[21] D. Birmingham, I. Sachs, and S. N. Solodukhin, "Relaxation in conformal field theory, Hawking-Page transition, and quasinormal/normal modes," Phys. Rev. D67 (2003) 104026, hep-th/0212308. 